\title{
Host Modulation in the Management of Endodontic Periodontic Lesions - A Report of 3 Cases
}

\author{
Afaf Zia ${ }^{1}$, Afshan Bey², Syed Mukhtar-un-Nisar Andrabi3 ${ }^{3}$, \\ Saif Khan ${ }^{4}$, Masood Khan ${ }^{5}$
}

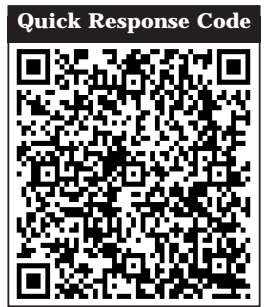

doi: 10.5866/2014.631637

${ }^{1 \& 4}$ Assistant Professor

2Professor

Department of Periodontology, Dr Z A Dental College, Aligarh Muslim University, Aligarh, India.

${ }^{3}$ Assistant Professor, Department of Conservative and Endodontics, Dr Z A Dental College, Aligarh Muslim University, Aligarh, India.

${ }^{5}$ Assistant Professor, Department of Oral Diagnosis \& Oral Pathology, Dr Z A Dental College, Aligarh

Muslim University, Aligarh, India.

\section{Article Info:}

Received: April 9, 2014

Review Completed: May 10, 2014

Accepted: J une 12, 2014

Available Online: October, 2014 (www.nacd.in) (C) NAD, 2014 - All rights reserved

\section{Email for correspondence:}

afafzia@gmail.com

\begin{abstract}
:
The present case series presents different patients with chief complaint of mobility in teeth where finally endo-perio involvement was seen. The endo-perio combined lesions generally show that there is communication between pulp and periodontal tissues because of common dental origin. Diagnosis in such cases is difficult and generally present problem in the management. All the cases were managed by non surgical endodontic therapy and host modulation was done. Local factors causing the lesion were removed. Clinical outcome after six and ninemonths werequite satisfactory. Combined endo-perio cases are challenging to the dentists and involve multidisciplinary involvement. To manage such cases, dentists have to rely on the unconventional techniques.
\end{abstract}

Key words: combined endo perio lesions, host modulation, non surgical therapy

\section{Introduction}

Combined endo-perio lesions are frequently encountered in dental clinics and are difficult to manage. Non surgical therapy like scaling and root planning and root canal therapy is the preferred choice of treatment. At times, surgical therapy like open flap debridement and regenerative techniques may be required. To overcome the need of surgery, a new therapy of host modulation is used nowadays. Host modulation is a recent concept where the proinflammatory mediators and destructive enzymes in the host are modified. Effective results have been seen when this therapy has been used in combination with the conventional techniques. ${ }^{1} \mathrm{We}$ report here three combined endo-periolesions where host modulation therapy has been used along with the conventional technique.

\section{Indian Journal of Dental Advancements}

Journal homepage: www. nacd. in 


\section{Case Reports}

\section{CASE 1}

A 25 year old female patient reported with slight pain and extreme mobility in right lower back teeth since 3 months. The patient was systemically healthy and there was no medical history contributory to the dental problem. Clinically, on probing 44 , a pocket around $11 \mathrm{~mm}$ was seen on both mesial and distal sides. Grade II mobility was present. Tooth was vital and the pain may be due to secondary endodontic invol vement. Plaque and calculus deposits were present and generalised pockets were also seen. IOPA X-ray revealed periapical radiolucency and excessive bone loss in relation to tooth 44. A diagnosis of chronic periodontitis was made. In 44 there was probably secondary endodontic involvement.

\section{CASE 2}

A 45 year old female patient came with the chief complaint of excessive mobility and slight pain in relation to lower front teeth. The patient had no contributory medical history to dental problem. Clinically, 31 and 42 teeth were highly mobile and gingival recession was seen. There was excessive plaque and calculus deposits and trauma from occlusion in the lower anterior incisors. Tooth 41 was extracted for periodontal reasons. Radiographically there was generalised bone loss. Teeth 31 and 42 showed immense bone loss and there was pain on percussion. This pain may be due to either trauma from occlusion or secondary endodontic involvement. Patient was diagnosed with chronic periodontitis.

\section{CASE 3}

A 24 Year old female came with chief complaint of mobility and slight pain in upper front teeth. Clinically, teeth 22 and 23 showed slight mobility and there was a pocket of $6 \mathrm{~mm}$ around 22 and 23 . Plaque and calculus deposits were seen. On percussion there was slight pain which may be due to secondary pulpal involvement. IOPA radiograph showed vertical bone resorption in relation to 23 . Diagnosis of chronic periodontitis was made with secondarily endodontic involvement in 22 and 23.

\section{Case management}

All the cases were managed with the same protocol. Treatment involved initially removal of all deposits by thorough scaling and root planning. Host modulation therapy was advocated from the day diagnosis was made i.e. they were all placed on subantimicrobial dose of doxycycline for 9 months twice daily. In case 2, trauma was removed in the lower anteriors by selective grinding. Three weeks after scaling and root planning, root canal therapy was done on all relevant teeth. At every recall visit oral hygiene instructions was reinforced. Follow up IOPA radiographs were taken at 6 months, 9 months and 1 year.

All the patients maintained hygiene and the rel evant involved teeth showed reduced mobility and patient were able to chew properly. After 6 months, it was seen in case $1 \& 2$ (Figure $1 b$ and $2 b$ ) that there was apparent bone fill. After 9 months radiographically, it was observed in all cases (Figure 1c, 2c and 3b) that there was bone fill and after a year marked bone fill was seen in all cases (F igure $1 d, 2 d$ and $3 c)$. On surgical entry in case 2 bone formation was seen after one year.

\section{Discussion}

The endo-perio lesion is a condition characterized by presence of endodontic and periodontal disease because of their common dental origin. The relationship between these two diseases was first described by Goldberg in $1964 .^{2}$ The deletorius effect of pulp on periodontium is well documented. The pulp can be invaded from periodontium through apical foramen, accessory canals and lateral canals. ${ }^{3,4}$ Combined endodonticperiodontic lesions present a great dilemma to the dentist as to what should be the approach of treatment. Such cases are generally managed by both non surgical and surgical phase. Non surgical phase includes thorough scaling and root planning and root canal treatment after a period of three months. Surgical phase involves better cleaning of root surfaces and filling of the advanced resorbed defects with grafts and membranes.

The present study reports three cases of primarily periodontal involvement with secondary endodontic involvement and their management. In the present cases non surgical therapy was used. Instead of surgically opening the defects and filling with bone fill material, the concept of host modulation was utilised. Periodontal diseases are initiated in presence of periodontopathic micro- 


\section{CASE 1}
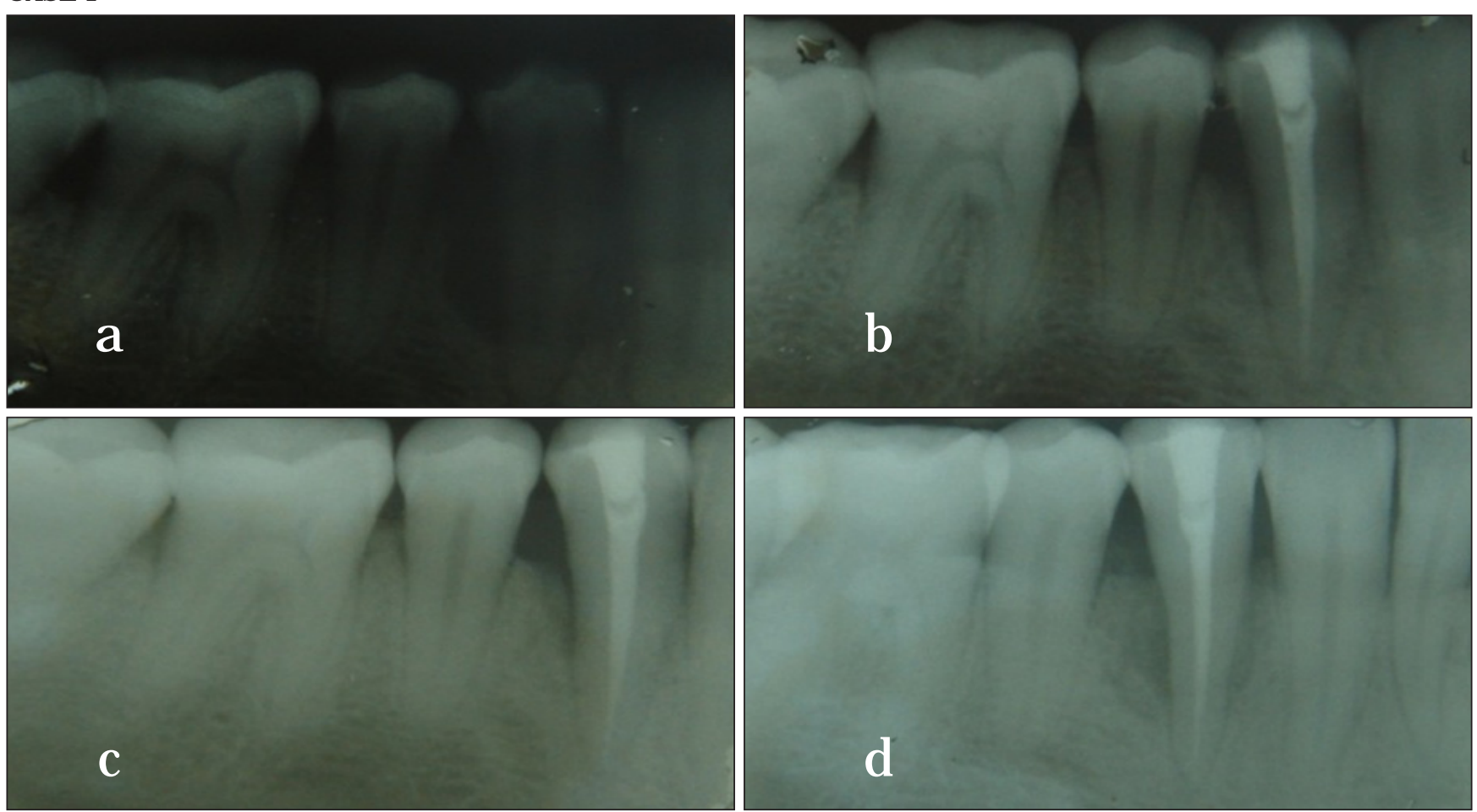

Figure 1: Case 1 - IOPA X-ray at a) baseline b) 6 months c) 9 months d) one year

\section{CASE 2}
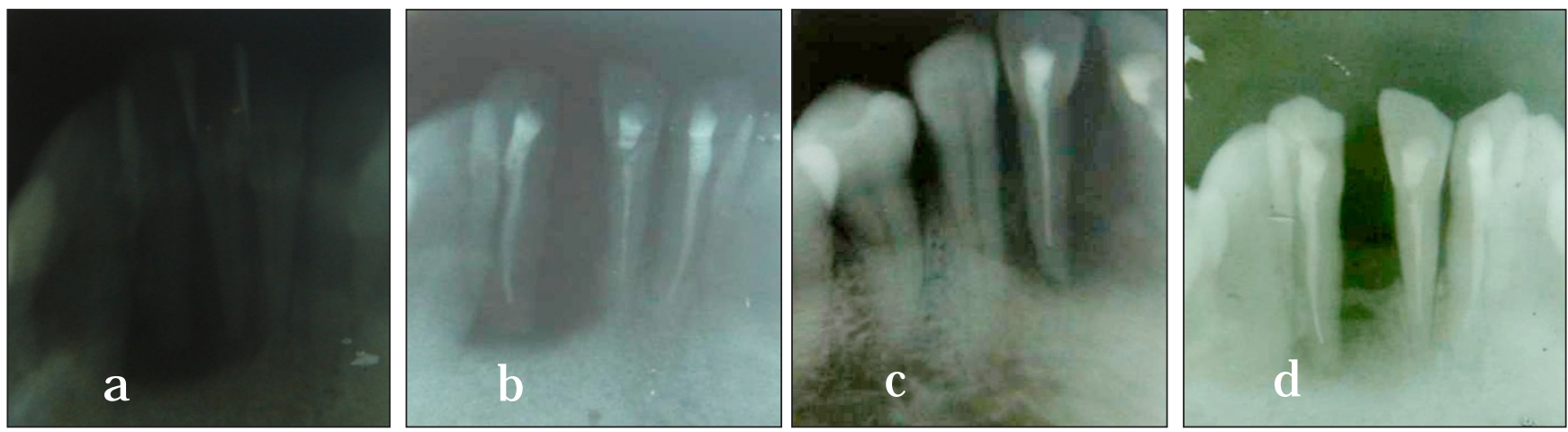

Figure 2: Case 2 - IOPA X-ray at a) baseline b) 6 months c) 9 months d) one year

CASE 3
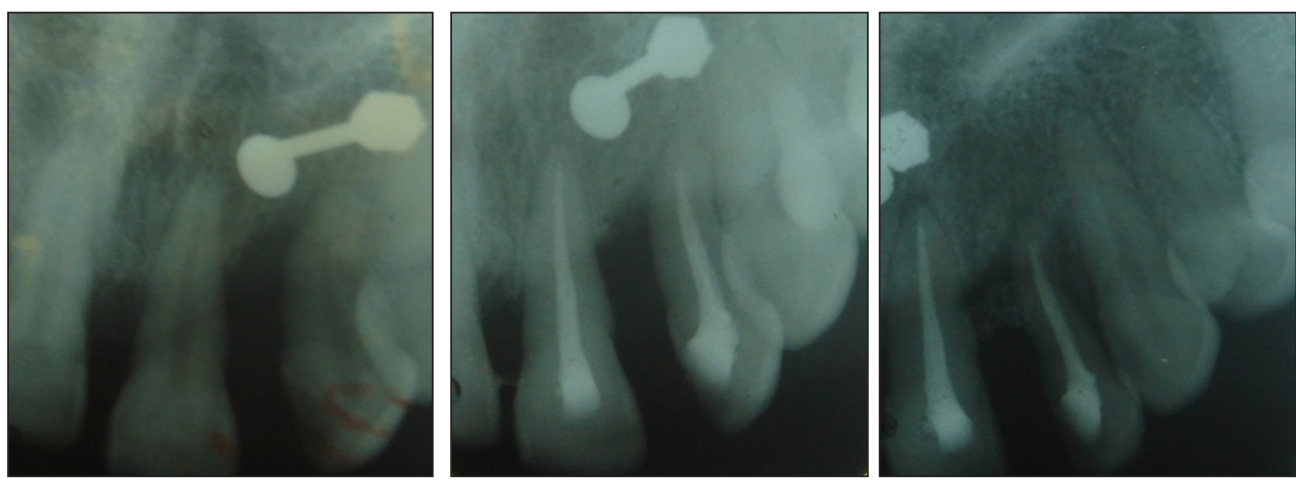

Figure 3: Case 1 - IOPA X-ray at a) baseline b) 6 months c) 9 months d) one year 
organisms and a susceptible host. Host modulation means modifying or modulating destructive or damaging aspects of the inflammatory host response that develops in the periodontal tissues as a result of the chronic challenge presented by the subgingival bacterial plaque. ${ }^{5}$ In certain situations conventional therapy may not result in the desired results. In such cases if one additionally modulates the host response, effective results can be gained. Hence, started host modulation therapy was started in all the three cases from the beginning of the treatment as an adjunct to conventional therapy. In all cases, there was primarily periodontal problem associated with secondary endodontic involvement.

After scaling and root planning in all the cases, there was slight decrease in mobility of teeth. In case 2, mobility was reduced immensely after removing the trauma. Patients were placed on maintenance therapy to evaluate their efficacy in maintaining oral hygiene. Root canal therapy was done after three months. IOPA radiograph after 6 months, showed slight bone fill in all the defects around the root canal treated teeth. Radiograph after 9 months showed immense bone fill. This regeneration may be due to combined effect of root canal therapy and host modulation. After 12 months, in all cases bone regeneration was seen to a greater extent. This bone formation may be due to decreased inflammation as a result of decreased microbial load by scaling and root planning. Also root canal therapy prevents the spread of inflammatory mediators from the pocket. Doxycycline as a host modulating agent inhibits host connective tissue breakdown through different mechanisms. Doxycycline at subantimicrobial doses inhibits the activity of matrixmetalloproteinases (MMPs), which are capable of degrading various extracellular molecules including collagen. ${ }^{6}$ In the connective tissue, doxycycline reduces osteoclast activity and bone resorption, blocks osteoclast MMP and stimulates osteoblasts activity and bone formation. Subantimicrobial dose of doxycycline has the advantage of being safe because of low plasma concentration (peak concentrations of 0.7-0.8 $\mu \mathrm{g} \mathrm{D}$ $\mathrm{ml}$ and steady- state concentrations of approximately $0.4 \mu \mathrm{g} \mathrm{D} \mathrm{ml}){ }^{7}$ No evidence of anitibiotic resistance have been identified in any of thestudies that have investigated this issuetill date. To our knowledge, this is one of the first case reports where endo-perio lesions are managed by host modulation.

\section{Conclusion}

In light of the treatment responses observed in this study, the healing potential of periodontal tissue, loss due to severe periodontitis treated with non-surgical and adjunctive therapy for host modulation requires further investigation. The host modulation appears to be an impetus for impressive clinical responses to non-surgical therapy. There is little information available on many host modulating therapies related to periodontitis and scarce data on the application of the host modulation therapy. Future devel opments in relation to subantimicrobial dose doxycycline will include modified-release formulations that achieve sustained plasma concentrations of doxycycline over 24 hours, but only require once per day dosing, thereby improving patient compliance. Thus, they should form part of an integrated treatment approach, together with hygiene therapy, plaque control, root surface instrumentation, maintenance care and risk factor modification.

\section{References}

1. Caton J G, Ciancio SG, Blieden TM, Bradshaw M, Crout RJ , Hefti AF, Massaro J M, Polson AM, Thomas J, Walker C. Treatment with subantimicrobial dose doxycycline improves the efficacy of scaling and root planing in patients with adult periodontitis. J Periodontol 2000; 71: 521-532.

2. Simring $M$, Goldberg $M$. The pulpal pocket approach: retrograde periodontitis. J . Periodontol 1964; 35: 22-48

3. Adriaens PA, DeBoever J A, LoescheWJ . Bacterial invasion in root cementum and radicular dentin of periodontally diseased teeth in humans. A reservoir of periodontopathic bacteria. J . Periodontol 1988; 59: 222-230

4. Czarnecki RT, Schilder H. A histologic evaluation of the human pulp in teeth with varying degrees of periodontal disease. J . Endod 1979; 5: 242-253

5. Preshaw PM. Host response modulation in periodontics. Periodontology 2008, 48:92-110

6. Birkedal-Hansen $\mathrm{H}$. Role of matrix metalloproteinases in human periodontal diseases. J Periodontol 1993; 64: 474484.

7. Walker C, Thomas J, Nango S, Lennon J, Wetzel J , Powala C. Long-term treatment with subantimicrobial dose doxycycline exerts no antibacterial effect on the subgingival microflora associated with adult periodontitis. J Periodontol 2000; 71: 1465-1471. 